\title{
SOME FUNCTIONS ON THE SET OF TRIANGLES OR QUADRANGLES
}

\author{
By SHUKICHI TANNO \\ Dedicated to Professor Shigeru Ishihara on his 60 th Birthday
}

\section{Introduction.}

Let $M=\left(A_{1} A_{2} \cdots A_{k}\right)$ be a polygon on a Euclidean 2-space $E^{2}$ with the canonical coordinate system $(x, y)$, and let $P=(x, y)$ be an arbitrary point of $E^{2}$. We define a function $f(x, y)=f_{M}(P)$ corresponding to $M$ by

$$
f_{M}(P)=\sum_{\imath=1}^{k}\left|\Delta P A_{\imath} A_{\imath+1}\right|^{2},
$$

where $A_{k+1}=A_{1}$ and $|\triangle P A B|$ denotes the area of the triangle determined by three points $P, A$ and $B$. Now we define $F(M)$ by

$$
F(M)=\int e^{-4 r^{2} f(x, y)} d E^{2},
$$

where $r$ denotes a non-zero real number. For a triangle $M$ we get the following equality :

$$
F(M)=\frac{\pi}{2 \sqrt{ } 3 r^{2} S(M)} e^{-4 r^{2} S(M)^{2} / 3},
$$

where $S(M)$ denotes the area of $M$ (cf. Theorem 2.1). The main result of this paper is the following (cf. Theorem 3.1.).

Theorem A. Let $M$ be a quadrangle on $E^{2}$. Then

$$
F(M) \leqq \frac{\pi}{2 r^{2} S(M)} e^{-r^{2} S(M)^{2}}
$$

holds, where the equality holds if and only if $M$ is a parallelogram. Therefore, for any parallelogram $M$ of the fixed area $S=S(M), F(M)$ is independent of the shape of $M$.

Let $Q=(x, y, z)$ be a point of a Euclidean 3 -space $E^{3}$ and define a function $g(x, y, z)=g_{M}(P)$ corresponding to a polygon $M$ by

Received May 4, 1982 


$$
g_{M}(P)=\sum_{\imath=1}^{k}\left|\Delta Q A_{\imath} A_{\imath+1}\right|^{2}
$$

Then we get a function $G(M)$ similarly (cf. §4).

The author is grateful to Professor Yoichiro Takahashi for introducing us the function $G(M)$ for quadrangles $M$.

\section{The axis of a triangle.}

Let $M=\triangle A B C$ be a triangle on a Euclidean 2-space $E^{2}$. We can assume that $M$ is placed so that the origin is the center of gravity of $M$ and $A=\left(0, b_{1}\right)$, $B=\left(-a, b_{2}\right)$ and $C=\left(a, b_{3}\right)$ with $a>0$. Then

$$
b_{1}+b_{2}+b_{3}=0 \text {. }
$$

The equations of lines are given by

$$
\begin{aligned}
& A B:\left(b_{1}-b_{2}\right) x-a y+a b_{1}=0, \\
& B C:\left(b_{2}-b_{3}\right) x+2 a y-a\left(b_{2}+b_{3}\right)=0, \\
& C A:\left(b_{1}-b_{3}\right) x+a y-a b_{1}=0 .
\end{aligned}
$$

Then, for a point $P=(x, y)$ of $E^{2}$ we obtain

$$
\begin{aligned}
4 f_{M}(P)= & 4\left(|\triangle P A B|^{2}+|\triangle P B C|^{2}+|\triangle P C A|^{2}\right) \\
= & {\left[\left(b_{1}-b_{2}\right) x-a y+a b_{1}\right]^{2}+\left[\left(b_{2}-b_{3}\right) x+2 a y+a b_{1}\right]^{2} } \\
& +\left[\left(b_{1}-b_{3}\right) x+a y-a b_{1}\right]^{2} \\
= & 6\left[\left(b_{1}^{2}+b_{1} b_{2}+b_{2}^{2}\right) x^{2}+a\left(b_{1}+2 b_{2}\right) x y+a^{2} y^{2}\right]+3 a^{2} b_{1}^{2} .
\end{aligned}
$$

If $b_{1}+2 b_{2} \neq 0$, we define an orthonormal base $\left(e_{1}, e_{2}\right)$ by $e_{1}=\left(e_{11}, e_{12}\right)$ and $e_{2}=\left(e_{21}, e_{22}\right)$, where

$$
\begin{aligned}
e_{12} / e_{11} & =\left(a^{2}-b_{1}^{2}-b_{1} b_{2}-b_{2}^{2}+\theta\right) / a\left(b_{1}+2 b_{2}\right), \\
e_{22} / e_{21} & =\left(a^{2}-b_{1}^{2}-b_{1} b_{2}-b_{2}^{2}-\theta\right) / a\left(b_{1}+2 b_{2}\right), \\
\theta & =\left[\left(a^{2}+b_{1}^{2}+b_{1} b_{2}+b_{2}^{2}\right)^{2}-3 a^{2} b_{1}^{2}\right]^{1 / 2} .
\end{aligned}
$$

Let $\left(x^{*}, y^{*}\right)$ be a new coordinate system with respect to $\left(e_{1}, e_{2}\right)$. Then we obtain

$$
4 f_{M}(P)=3\left(\lambda_{1} x^{*^{2}}+\lambda_{2} y^{*^{2}}\right)+3 a^{2} b_{1}^{2}
$$

where

$$
\lambda_{1}, \lambda_{2}=a^{2}+b_{1}^{2}+b_{1} b_{2}+b_{2}^{2} \pm \theta .
$$

If $b_{1}+2 b_{2}=0$, then $\lambda_{1}=3 b_{2}^{2}$ and $\lambda_{2}=a^{2}$ in (2.1) with respect to $(x, y)$. In any case, we obtain $\lambda_{1} \lambda_{2}=3 a^{2} b_{1}^{2}$. The area $S(M)$ of $M$ is given by $S(M)=3 a b_{1} / 2$ 
and hence $3 a^{2} b_{1}^{2}=4 S(M)^{2} / 3$. Therefore

$$
\begin{aligned}
\int e^{-4 r^{2} f(x, y)} d E^{2} & =\int e^{-3 r^{2} \lambda_{1} x^{2}} d x \int e^{-3 r^{2} \lambda_{2} y^{2}} d y e^{-3 r^{2} a^{2} b_{1}^{2}} \\
& =\frac{\pi}{3 r^{2}\left(\lambda_{1} \lambda_{2}\right)^{1 / 2}} e^{-4 r^{2} S(M)^{2} / 3}
\end{aligned}
$$

Thus, we get

THEOREM 2.1. For a triangle $M$ on $E^{2}$,

$$
F(M)=\frac{\pi}{2 \sqrt{3} \frac{\pi}{r^{2} S(M)}} e^{-4 r^{2} S(M) 2 / 3}
$$

holds, i.e., $F(M)$ depends only on the area of $M$.

We may call $e_{1}$-direction ( $e_{2}$-direction, resp.) the axis of the triangle $\triangle A B C$. If $\triangle A B C$ is an equilateral triangle, then $b_{1}=-2 b_{2}=-2 b_{3}, a^{2}=3 b_{2}^{2}$ and $\theta=0$ hold, and hence $e_{1}$ is not definite. In this case any direction through the center of gravity of $\triangle A B C$ is an axis.

A geometric meaning of the axes of a triangle is (2.1). It is an open question if there are other geometric meanings of axes of a triangle.

\section{3. $F(M)$ for a quadrangle.}

Let $M=(A B C D)$ be a quadrangle on $E^{2}$. We can assume that $M$ is placed so that

$$
\begin{array}{ll}
A=(-a, 0), & B=(e,-d), \\
C=(b, 0), & D=(0, c),
\end{array}
$$

where $b>0, a+b>0$ and $c>0$.

The equations of lines are given by

$$
\begin{aligned}
& A B: d x+(a+e) y+a d=0, \\
& B C: d x-(b-e) y-b d=0, \\
& C D: c x+b y-b c=0, \\
& D A: c x-a y+a c=0 .
\end{aligned}
$$

Then, for a point $P=(x, y)$ of $E^{2}$ we obtain

$$
\begin{aligned}
4 f_{M}(P)= & {[d x+(a+e) y+a d]^{2}+[d x-(b-e) y-b d]^{2} } \\
& +[c x+b y-b c]^{2}+[c x-a y+a c]^{2}
\end{aligned}
$$


FUNCTIONS ON THE SET OF TRIANGLES OR QUADRANGLES

$$
\begin{aligned}
= & 2\left(c^{2}+d^{2}\right) x^{2}+2\left[a^{2}+b^{2}+e^{2}+(a-b) e\right] y^{2} \\
& +2[-(a-b)(c-d)+2 d e] x y+2(a-b)\left(c^{2}+d^{2}\right) x \\
& +2\left[-\left(a^{2}+b^{2}\right)(c-d)+(a-b) d e\right] y+\left(a^{2}+b^{2}\right)\left(c^{2}+d^{2}\right) .
\end{aligned}
$$

Putting $x=\bar{x}-\alpha, y=\bar{y}-\beta$, we get

where

$$
\begin{aligned}
4 f_{M}(P)= & 2\left(c^{2}+d^{2}\right) \bar{x}^{2}+2\left[a^{2}+b^{2}+e^{2}+(a-b) e\right] \bar{y}^{2} \\
& +2[-(a-b)(c-d)+2 d e] \overline{x y}+C(M),
\end{aligned}
$$

$$
\begin{aligned}
\alpha= & (a-b)\left(a^{2}+b^{2}\right)(c+d)^{2} / K+2(a-b) c^{2} e^{2} / K \\
& +\left[(a-b)^{2}\left(2 c^{2}+d^{2}+c d\right)+2\left(a^{2}+b^{2}\right)(c-d) d\right] e / K, \\
\beta= & -(a+b)^{2}\left(c^{2}+d^{2}\right)(c-d) / K, \\
K= & 3\left(a^{2}+b^{2}\right)\left(c^{2}+d^{2}\right)+2\left[a b(c-d)^{2}+\left(a^{2}+b^{2}\right) c d\right] \\
& +4(a-b) c(c+d) e+4 c^{2} e^{2} \\
= & 2(a+b)^{2}\left(c^{2}+d^{2}\right)+[2 c e+(a-b)(c+d)]^{2}>0, \\
C(M)= & -\alpha(a-b)\left(c^{2}+d^{2}\right)-\beta\left[-\left(a^{2}+b^{2}\right)(c-d)+(a-b) d e\right] \\
& +\left(a^{2}+b^{2}\right)\left(c^{2}+d^{2}\right) \\
= & S(M)^{2}+T(M),
\end{aligned}
$$

where $S(M)=(a+b)(c+d) / 2$ is the area of $M$ and

$$
\begin{aligned}
T(M)= & (a+b)^{2}(c-d)^{2} c^{2} e^{2} / K+(a-b)(a+b)^{2} c(c-d)\left(c^{2}-d^{2}\right) e / K \\
& +(1 / 4)(a-b)^{2}(a+b)^{2}(c-d)^{2}(c+d)^{2} / K \\
= & (a+b)(c-d)^{2}[c e+(1 / 2)(a-b)(c+d)]^{2} / K \geqq 0 .
\end{aligned}
$$

$T(M)=0$ holds if and only if $c=d$ or $2 c e=-(a-b)(c+d)$. The first three terms of the right hand side of (3.1) is written as

where $\lambda_{1}$ and $\lambda_{2}$ are given by

$$
\lambda_{1} x^{2}+\lambda_{2} y^{*^{2}}
$$

$$
\begin{aligned}
\lambda_{1}, \lambda_{2}= & a^{2}+b^{2}+c^{2}+d^{2}+e^{2}+(a-b) e \pm L, \\
L^{2}=[ & \left.a^{2}+b^{2}+c^{2}+d^{2}+e^{2}+(a-b) e\right]^{2} \\
& -\left[3\left(a^{2}+b^{2}\right)\left(c^{2}+d^{2}\right)+2 a b\left(c^{2}+d^{2}\right)+2(a-b)^{2} c d\right. \\
& \left.+4(a-b)(c+d) c e+4 c^{2} e^{2}\right] .
\end{aligned}
$$

Consequently, 
where

$$
\begin{aligned}
\lambda_{1} \lambda_{2}= & 3\left(a^{2}+b^{2}\right)\left(c^{2}+d^{2}\right)+2 a b\left(c^{2}+d^{2}\right)+2(a-b)^{2} c d \\
& +4(a-b)(c+d) c e+4 c^{2} e^{2} \\
= & 4 S(M)^{2}+R(M),
\end{aligned}
$$

$$
R(M)=[2 c e+(a-b)(c+d)]^{2}+(a+b)^{2}(c-d)^{2} \geqq 0 .
$$

$R(M)=0$ holds if and only if $c=d$ and $c=b-a$, i. e., $M$ is a parallelogram.

Summarizing the above we obtain the following.

THEOREM 3.1. Let $M$ be a quadrangle on $E^{2}$. Let $S(M)$ denote the area of $M$ and define $T(M)$ and $R(M)$ by (3.2) and (3.3), respectively. Then

$$
\begin{aligned}
\int e^{-4 r^{2} f(x, y)} d E^{2} & =-\frac{\pi}{r^{2}\left[4 S(M)^{2}+R(M)\right]^{1 / 2}} e^{-r^{2}\left(S(M)^{2}+T(M)\right)} \\
& \leqq \frac{\pi}{2 r^{2} S(M)} e^{-r^{2} S(M)^{2}}
\end{aligned}
$$

where $T(M)=R(M)=0$ holds if and only if $M$ is a parallelogram.

Corollary 3.2. Among parallelograms on $E^{2}, F(M)$ does not depend on the shape of $M$, but only on the area of $M$.

\section{4. $G(M)$ for $M$ on $E^{2}$ in $E^{3}$.}

Let $M=(A B C D)$ be a quadrangle on $E^{2}$ and let $Q=(x, y, z)$ be a point of a Euclidean 3-space $E^{3}$. By $P=(x, y)$ we denote the image of $Q$ by the natural projection: $E^{3} \rightarrow E^{2}$. Then

$$
4|\triangle Q A B|^{2}=|A B|^{2} z^{2}+4|\triangle P A B|^{2}
$$

holds, where $|A B|$ denotes the length of the segment $A B$. We define functions $g(x, y, z)=g_{M}(Q)$ and $G(M)$ by

$$
\begin{aligned}
g_{M}(Q) & =|\triangle Q A B|^{2}+|\triangle Q B C|^{2}+|\triangle Q C D|^{2}+|\triangle Q D A|^{2}, \\
G(M) & =\int e^{-4 r^{2} g(x, y, z)} d E^{3} .
\end{aligned}
$$

Then

where we have put

$$
\begin{aligned}
G(M) & =\left(\int e^{-r^{2} V(M) z^{2}} d z\right) F(M) \\
& =\sqrt{\pi} r^{-1} V(M)^{-1 / 2} F(M),
\end{aligned}
$$


FUNCTIONS ON THE SET OF TRIANGLES OR QUADRANGLES

$$
\begin{aligned}
V(M) & =|A B|^{2}+|B C|^{2}+|C D|^{2}+|D A|^{2} \\
& =2\left[a^{2}+b^{2}+c^{2}+d^{2}+e^{2}+(a-b) e\right] \\
& =4 S(M)+U(M), \\
2 U(M)= & 2(a-d)^{2}+2(b-d)^{2}+(a+b-2 c)^{2}+(a-b+2 e)^{2} \geqq 0,
\end{aligned}
$$

where $a, b, c, d, e, S(M)$ and $F(M)$ are ones used in $\S 3$. $U(M)=0$ holds if and only if $M$ is a square. Applying Theorem 3.1, we obtain

Theorem 4.1. Let $M$ be a quadrangle on $E^{2}$. Then $G(M)$ satisfies the following.

$$
\begin{aligned}
G(M) & =\frac{\pi^{3 / 2}}{r^{3}\left[(4 S(M)+U(M))\left(4 S(M)^{2}+R(M)\right]^{1 / 2}\right.} e^{-r^{2}\left(S(M)^{2}+T(M)\right)} \\
& \leqq \frac{\pi^{3 / 2}}{4 r^{3} S(M)^{3 / 2}} e^{-r^{2} S(M)^{2}}
\end{aligned}
$$

where $R(M), S(M)$ and $T(M)$ are defined in $\S 3$, and $R(M)=T(M)=U(M)=0$ holds if and only if $M$ is a square.

Department of Mathematics

Tokyo Institute of Technology 